3-D route-planning support for wayfinding

\title{
3-D route-planning support for navigation in a complex indoor environment
}

Abstract. Three-dimensional (3-D) route-planning support offers a promising solution to overcome problems with wayfinding in complex indoor environments. An experiment was conducted to test the effect of 3-D route-planning support in a realistic 'live' setting, a large hospital building, during normal operation. Forty participants performed navigation tasks either with $(n=20)$ or without $(n=20) 3-\mathrm{D}$ Formatted: Font: Italic route-planning support. Support resulted in faster navigation, more use of artwork Formatted: Font: Italic specifically installed to aid wayfinding, fewer navigation errors, less disorientation and less anxiety. In addition, participants used different strategies for wayfinding: without navigation support they used signs and route colour, but with navigation support they used not only the artwork, but also existing furniture and other landmarks. The acceptance of 3-D route-planning support was high. Overall, the results support the value of 3-D route-planning support.

Keywords: wayfinding; navigation; indoor environment; 3-D environment; visualization

\section{Introduction}

\subsection{Navigation in complex buildings}

When using navigating unfamiliar complex buildings, such as hospitals, people experience problems in navigation and disorientation, not knowing where they are and how to get to their destination (Rooke et al., 2009; Vilar et al., 2012). Work in environmental psychology has identified various negative consequences of disorientation. These include stress and frustration of visitors (resulting in negative physical and psychological effects), visitors not being able to find their way (resulting 


\section{3-D route-planning support for wayfinding}

in late appointments, loss of reputation, time, opportunities and money), and consequences for organisations (such as disruption of hospital schedules, loss of revenue from customers who do not comearrive at their destination, and time and effort to direct and escort visitors to their destinations) (Carpman \& Grant, 2002). Route-planning and support for route-planning may help in overcoming some of the problems people experience in navigating vast, ${ }_{2}$ busy indoor environments, such as large hospitals, by allowing them to develop spatial knowledge that is useful in finding their way to a particular destination.

\subsection{Navigation and route-planning}

Navigation has two components: wayfinding (the cognitive component) and motion (the motoric component) (Barken \& PetersonDarken \& Peterson, 2002, 2002). The current research is mainly concerned with wayfinding and in particular with routeplanning to a target destination. More generally, wayfinding does not only involve planning before one walks a route, but during navigation wayfinding and motion are closely linked (Darken \& Peterson, 2002). As an essential element of wayfinding, people develop and use a spatial representation ('cognitive map'; Tolman, 1948) of their environment. Three hierarchical levels of spatial knowledge are distinguished in the development of a cognitive map: landmark knowledge, route knowledge and survey knowledge (Downs \& Stea, 1977Darken \& Sibert, 1996). Route-planning may assist people in developing their knowledge of landmarks (salient objects or structures and other visual features of the environment, as individual items), in advance of walking a route in the physical world. In addition, route-planning may help people to develop route knowledge (regarding a series of landmarks with turning information that connect to form a route from a starting point to a destination) and survey knowledge (concerning the spatial configuration of an environment, 
3-D route-planning support for wayfinding

enabling people to generate new routes). Models of navigation (e.g. Jul \& Furnas, 1997, cited in Darken \& Peterson, 2002) clarify the role of these different types of knowledge in the process of navigation.

\subsection{Route-planning support}

In order to study and solve problems in wayfinding it is important to consider the wayfinding system (Carpman \& Grant, 2002). The following three components are involved in and influence the quality of wayfinding and should be considered in the design of wayfinding systems: people (e.g. visitors, patients and staff), design elements (e.g. the structural layout of a building) and operational elements (e.g. indoor signage system).

Regarding people, research has demonstrated that background knowledge influences decisions in wayfinding (Cornell \& Heth, 2000; Frankenstein et al., 2012). Furthermore, people with a good self-reported sense of direction rate survey descriptions higher, and spatial (mental-rotation) ability is negatively correlated with wayfinding errors and positively with rating of survey directions (Padgitt \& Hund, 2012). On the subject of design elements, research has demonstrated that building complexity has a negative effect on navigation efficiency, presumably due to a limitation of spatial memory (Stankiewicz et al., 2001). Furthermore, spatial differentiation of an indoor environment aids the development of a cognitive map $(X u$ et al., 2009). Other research has demonstrated that width and brightness of corridor intersections influences people's preference regarding egress in emergency situations; when they arrive choice point between two corridors they are more likely to choose the corridor that is wider and brighter than one that is not -(Vilar et al., 2013). As regards operational elements, research has modelled the effect of 
3-D route-planning support for wayfinding

improved signage on the use of signage during an evacuation (Xie et al., 2012). The current study examines an operational element - route-planning support - and its (positive) effects on people's wayfinding in a complex hospital environment.

The current research focuses on dynamic 3-D representations to aid route-planning. The use of 3-D representations during route-planning could help people in more effectively constructing a cognitive representation of the to-be-navigated environment (Darken \& Peterson, 2002) in terms of the three types of spatial knowledge. Particular features of dynamic 3-D representations that may contribute to this include the following: they can provide an ego-centric perspective, high-

fidelity information regarding the environment and richer or more detailed information for the landmarks. In particular, they allow the presentation to highlight landmarks at the expense of less salient elements of the environment in order to facilitate the encoding of landmarks in relation to the environment. Therefore, a promising solution to the problems caused by wayfinding in complex buildings is offered by 3-D route-planning, with the aim of improving people's navigation performance (e.g. reducing time-on-task and errors) and experience (e.g., reducing disorientation and anxiety). These representations can be advantageous over other representations, such as maps. For example, people may study a print map in advance of going on a journey. However, maps are relatively abstract representations of the real world and people may have difficulty in using these effectively possibly because they require additional mental effort in using the spatial information that is being presented (Münzer \& Stahl, 2011). Wright et al. (2010) studied the provision of route information in a hospital setting, where a 'static' route to selected destinations was presented in two dimensions. Therefore, planning involved passively receiving static information. Moreover, very limited data collection was done and no experimental or 


\section{3-D route-planning support for wayfinding}

other comparison was made between various types of route-planning support or between support and no support.

Münzer and Stahl (2011) investigated the effect of route-planning support with three types of route visualization in an indoor university environment. The results showed an advantage in wayfinding of animated over static egocentric visualizations (pictures from a first-person view, with decision points), but no difference between allocentric (map) and egocentric visualizationsegocentric visualizations. In addition, wayfinding performance was correlated with gender, visual-spatial abilities and egocentric wayfinding strategy (both mediators of gender); these findings are consistent with Lawton's (1996) observation that gender and orientation strategies are associated with wayfinding performance, although findings regarding gender are not consistent across research studies. Limitations of this work are that only one route was tested and, although the building was considered complex, it was relatively small compared to, for instance, a large hospital.

Although the usability and utility of interactive systems is important for their success, potential benefits in improved task performance will be lost if acceptance is low (Venkatesh et al., 2007). Therefore, it is critical that the acceptance of new technology is also established. In particular, perceived usability, perceived usefulness and intention to use are important indicators of acceptance. These may be linked to aspects of task performance, for example wayfinding behaviour. However, although the correlation between technology acceptance and task performance is substantial over studies, thisere is not universal (Rientjes et al., 2014). Moreover, more recently user-experience (e.g. disorientation) has been identified as a precursor of technology acceptance (Saadé \& Otrakji, 2007). In sum, 


\section{3-D route-planning support for wayfinding}

it is crucial that potential users' acceptance of any new technology is established as this is essential for its success and acceptance may be linked to task performance and experience.

It is important to note that route-planning (the subject of this study) fundamentally differs from navigation systems that provide online wayfinding support (Fallah et al., 2013). Examples of the latter include GPS-based car navigation systems and GPSbased pedestrian-wayfinding systems. Although online support can be useful, it relies on providing instructions and users following instructions rather than supporting users in building a cognitive map of their to-be-navigated environment, which may be facilitated better by route-planning support. ${ }^{1}$ In addition, indoor navigation systems suffer from significant problems of cost, usability and accuracy (Fallah et al., 2013).

Although the usability and utility of interactive systems is important for their success, potential benefits in improved task performance will be lost if acceptance is low (Venkatesh et al., 2007). Therefore, it is critical that the acceptance of new technology is also established. In particular, perceived usability, perceived usefulness and intention to use are important indicators of acceptance. These may be linked to aspects of task performance, for example wayfinding behaviour. However, although the correlation between technology acceptance and task performance is substantial over studies, there is not universal (Rientjes et al., 2014). Aoreover, more recently user-experience (e.g. disorientation) has been identified as a precursor of technology acceptance (Saadé \& Otrakji, 2007). In sum, it is crucial

\footnotetext{
1. The use of landmarks can also facilitate navigation in online navigation support for pedestrians (e.g. Goodman et al., 2005) or drivers (Lavie \& Oron-Gilad, 2013), but here their function is not to aid users in building a cognitive map, but to give directions or information.
} 
3-D route-planning support for wayfinding

that potential users' acceptance of any new technology is established as this is essential for its success and acceptance may be linked to task performance and experience.

\subsection{Rationale and current study}

There is a lack of research investigating the effect of 3-D representations in routeplanning on navigation in complex buildings (however, see Münzer \& Stahl, 2011). In particular, there is a lack of research examining the advantage of 3-D representations as additional support over and above existing wayfinding support offered in large complex public buildings, and studying navigation of multiple routes and the role of landmarks.

Therefore and given that 3-D route-planning offers a promising solution to problems caused by wayfinding in complex buildings, the aim of the current study is to establish the facilitating effect of 3-D route-planning support on wayfinding in a large complex public indoor environment using multiple routes, the role of landmarks and system acceptance. Accordingly, this research addresses two research questions. Research Question 1: does 3-D route-planning support enhance wayfinding behaviour and experience in complex buildings? As discussed in Section 1.3, this type of support can aid in the development of spatial knowledge that people can employ in wayfinding. Research Question 2: what is the level of acceptance for 3-D route-planning support? As a result of facilitating wayfinding and people's experience of wayfinding, this support may be well accepted. The research was conducted in a 'live'large hospital during normal operation with many staff, many patients and many visitors, adding to the ecological validity of this work. The results contribute to knowledge regarding wayfinding in complex environments and support 
3-D route-planning support for wayfinding

for this activity, and provide evidence for practitioners to aid in the design of these environments and of support for wayfinding.

\section{Method}

\subsection{Design}

An independent-measures experimental between-subjects design was used with the independent variable route-planning support. 3-D route-planning support was provided in the experimental condition $(n=20)$, but not in the control condition $(n=$ 20). In both conditions, the existing static wayfinding system (see Section 2.3) and the hospital environment were enhanced with additional landmarks -specifically designed to aid wayfinding for the following reason. The concept behind the 3-D wayfinding system that was used is to capitalise on salient landmarks that have been deliberately placed in a built indoor environment to aid wayfinding, by including these in the 3-D model that the system visualizes to aid route-planning. This way, the system can aid the development of landmark knowledge during route planning. Dependent variables were time-on-task, errors and hesitations in wayfinding, disorientation, use of landmarks and anxiety. In addition, the acceptance of the 3-D route-planning support was measured in the experimental condition.

\subsection{Participants}

Forty participants ( 25 male) were recruited on a voluntary basis among students at ***** university (name of establishment removed to facilitate masked review) and were paid $£ 5$ for taking part. Inclusion criteria were speaking English, being aged 18 or over, not having a disability and not having visited ${ }^{* * * * *}$ hospital (name of 
3-D route-planning support for wayfinding

establishment removed to facilitate masked review) before. Potential participants were identified through university e-mail lists.

\subsection{Indoor environment, materials and equipment}

At the time of the research, ${ }^{\star \star * * *}$ hospital was a large hospital with 74 departments and wards as well as various facilities, including a three shops, a restaurant and three cafeterias $s_{-} \div$Mmore than 6000 staff worked in the hospital, and there were approximately 2400 patients per day and approximately the same number of visiting or accompanying relatives or friends. The size of the complex main hospital building (in addition to three relatively very-small buildings) was more than $100000 \mathrm{~m}^{2}$ and it had three floors, connected by stairs and lifts. The main building had nine different entrances. The wayfinding system in the hospital included plans posted on the corridor walls, signs to hospital departments and a system of four coloured routes (orange, blue, green and yellow) that were displayed in various places in the hospital.

Three routes were chosen, corresponding with three wayfinding tasks, to represent a variety of destinations that offered challenges in wayfinding through choice points (see Appendix). The length of the routes was $156 \mathrm{~m}, 172 \mathrm{~m}$ and $158 \mathrm{~m}$ for the three respective routes. A route's difficulty for wayfinding might be primarily negatively affected by its number of choice points, but also by positively by the frequency of signboards and maps with information indicating the route's destination (the more signs and maps, the more en-route support there is for wayfinding), the overlap with other routes (so wayfinders can apply their knowledge of a previous overlapping route to complete the current route) and the number of ways to reach the destination (e.g. the more solutions exist, the higher the chance of reaching the destination). 


\section{3-D route-planning support for wayfinding}

According to this objective characterisation of difficulty, Route 1 would be most difficult (eight choice points, $70 \%$ of signs and maps leading to destination, no overlap with other routes and one way from start to end), followed by Route 3 (six choice points, $80 \%$ of signs and maps, overlap with Route 2 and four alternative ways from start to end) and Route 2 (four choice points, $80 \%$ of signs and maps, overlap with Route 3 and five alternative ways from start to end).

For the purpose of the study, at strategically selected locations, nine additional large conspicuous landmarks $($ size: $1 \mathrm{~m}(\mathrm{~h}) \times 0.8 \mathrm{~m}(\mathrm{w})$ [Large pieces]; $0.6 \mathrm{~m}(\mathrm{~h}) \times 0.4 \mathrm{~m}(\mathrm{w})$ [small pieces]) were placed to further aid wayfinding (see Figure 1). The aim of route-planning support was to aid people in developing landmark- and route knowledge. Those who were not given route-planning support would still be able to see the landmarks, but the landmarks were not expected to facilitate their wayfinding. Although the provision of large conspicuous landmarks might be especially-particularly effective in 'featureless' indoor environments, route-planning would be expected to benefit from such conspicuous landmarks even in environments that already have furniture and less conspicuous artwork, because conspicuous landmarks would be more likely to attract wayfinders' attention and therefore be encoded in memory to develop landmark- and route knowledge. Insert Figure 1 about here.

A commercially available 3-D route-planning system, housed in a kiosk with a 19inch screen having a resolution of 1024 by 768 with a 19 -inch and a screen resolution of 1024 by 768 pixels, was used to represent the complex indoor environment of

hospital (see Figure 2). Through animation, the system showed a walkthrough of the route in 3-D from a fixed starting point to a destination selected by a user, 
3-D route-planning support for wayfinding

without control over navigation or playback. Animation, rather than self-paced 'virtual walking' (Author Citation 1), was used to make efficient use of route-planning time and to avoid disorientation and wayfinding errors during route-planning. The frame rate was 60 pictures per second. It took $107 \mathrm{~s}, 123 \mathrm{~s}$ and $87 \mathrm{~s}$ to present the three respective routes. Walking speed in the 3-D animation was set at $9.36 \mathrm{~km}$ per hour for efficient use of route-planning time. The system supported singledestination journeys from an entrance point to the hospital, as the most common expected use of the route-planning was reaching a range of single destinations from such a point.

Insert Figure 2 about here.

Existing instruments were used to test users' spatial ability (Vandenberg \& Kuse, 1978) and measure disorientation (7-point Likert scales, based on Ahuja \& Webster, 2001), acceptance (perceived ease of use, perceived usefulness and intention to use) of the route-planning support system (7-point Likert scales, based on Venkatesh et al., 2003) and state anxiety (4-point Likert scale, based on Abed et al., 2011, and Spielberger, 1983). Psychometric scales had good reliability, with Cronbach's alpha $>0.70$ (disorientation: 0.84 to 0.96 for different routes; perceived usefulness: 0.90 ; perceived ease of use: 0.88 ; intention to use: 0.93 ; pre-test anxiety: 0.75; post-test anxiety: 0.94). Therefore, mean scores were calculated on the scales and used in further data analysis. Errors in wayfinding were defined by enumeration, with the following categories: wrong turns, losing the way, going round in circles and walking past the route's destination. Hesitations occurred when a participant stopped walking for more than 10 seconds. 
3-D route-planning support for wayfinding

\subsection{Procedure}

In Stage 1, participants arrived at the main hospital reception, met the researcher, read the participant's information sheet and filled in the consent form. The researcher checked if inclusion criteria were met. In Stage 2, participants were assigned a research identifier. They were briefed about their task (and the use of the route-planning support system in the experimental condition). All participants were provided with general instructions about the navigation task (e.g., they should not ask questions during wayfinding, but they should indicate when they completed a task and work as quickly and efficiently as possible) and completed the anxiety questionnaire and the spatial-ability test. In Stage 3, participants completed three navigation tasks unaided, with tasks counterbalanced among participants. Before

\section{starting their journey on any task, participants in the experimental condition selected}

$\underline{\text { the destination on the system and then viewed the route to the destination as shown }}$

by the system. First, on the outwards journey, they had to find their way from the starting point to the particular task's destination. Then, on the return journey, they had to find their way back. After each journey they completed the disorientation scale. During each journey, the researcher recorded notes of observations on a printed copy of the route for the corresponding task. If a participant made an error, the researcher told the participant, but did not provide any directions. In Stage 4, participants answered questions about their use of artwork in the navigation tasks and completed post-use questionnaires (anxiety and technology acceptance [experimental group only]). In Stage 5, participants were debriefed and reimbursed for their participation. The experiment took about 25-30 minutes to complete in the experimental group, and about 40-45 minutes in the control condition. 


\section{3-D route-planning support for wayfinding}

\section{Results}

\subsection{Wayfinding behaviour and experience}

This section presents results related to Research Question 1. The results of additional exploratory analyses are presented to gain further insight into wayfinding behaviour in relation to wayfinding strategy and task difficulty. Results are reported for the outward journey of each task, as the route-planning system that was used supported the planning of outward journeys, but not return journeys.

Visual inspection of descriptives (see Table 1) shows $\underline{\text { indicates an apparent }}$ substantial advantage of 3-D route-planning support, in particular on time-on-task on all tasks, errors on Task 1 and Task 3 and disorientation on Task 1. In addition, there was a very substantial difference between the two conditions on reported using artwork in wayfinding: 19 out of 20 participants in the experimental condition reported using artwork, but only 2 out of 20 in the control condition. Furthermore, there appeared to be substantial differences between those who reported using of artwork and those who did not, with better outcomes for the former (see Table 2). From descriptives by gender, there was no apparent gender difference on any of the measures and statistical tests showed no difference on these variables or spatial ability.

Insert Table 1 and Table 2 about here.

Test results show a significant positive effect of route-planning support on time-ontask on Task 1, $t(38)=8.83, p<0.001, d=2.15^{2}$, Task $2, t(38)=6.11, p<0.001, d$

2 The effect size measure $d$ is defined as the difference between two group means divided by their (pooled) standard deviation (the number of standard deviations that two means [here experimental and control] are apart). For interpretation, effect size conventions are $0.2=$ small, $0.5=$ medium and 0.8 = large (Cohen,1988). 
3-D route-planning support for wayfinding

$=1.35$, and Task $3, t(38)=4.81, p<0.001, d=1.15$. There was also a significant positive effect in terms of reducing errors on Task $1, t(38)=-4.07, p<0.001, d$ $=-1.11$, and Task $3, t(38)=-2.70, p=0.01, d=-0.60$. The positive effect of routeplanning support in terms of reducing hesitations on Task 1 was approaching significance, $t(38)=-1.95, p=0.06, d=-0.54$. Route-planning support also had a positive effect in terms of reducing disorientation on Task $1, t(38)=-3.22, p<0.01$, $d=-0.84$. Furthermore, with route-planning support post-test anxiety was reduced, , $t(38)=-2.35, p<0.05, d=-0.84$. In addition, the effect of route-planning support on the reported use of artwork was significant, $\varphi=0.85, p<0.001$.

In turn, the reported use of artwork was associated with shorter time-on-task on all tasks $($ Task 1: $t(38)=5.94, p<0.001, d=1.82$; Task 2: $t(38)=4.22, p<0.001, d=$ 1.09; Task 3: $t(38)=4.23, p<0.001, d=1.08)$, fewer errors on Task $1, t(38)$ $=-3.18, p<0.01, d=-1.20$, fewer errors on Task $3, t(38)=-2.24, p<0.05, d$ $=-1.96$, and less disorientation on Task $1, t(38)=-3.32, p<0.01, d=-1.49$. The association of artwork with hesitations on Task 1 was approaching significance, $t$ $(38)=-1.80, p<0.10, d=-0.69$. Spatial ability was not related to route-planning support or the use of artwork, both $p<<0.05$.

Generally, it was observed that very few participants made use of the existing hospital signage system, for example plans posted on the corridor walls. Those in the control condition who did use these plans, completed their tasks with fewer errors. In addition, it was observed that the frequency of signs and maps leading to the destination (lower on Route 1 than on Routes 2 and 3) had an impact predominantly on participants' wayfinding on the control condition. Furthermore, it was found that experimental condition and participants' reported of use artwork were 
3-D route-planning support for wayfinding

negatively correlated with the observed use of signs $(\phi=-0.73$ and -0.70 ,

respectively, both $p<0.001)$ and route colour $(\phi=-0.46$ and -0.48 , respectively, both $p<0.01$ ), but positively correlated with observed use of existing furniture (e.g. a sofa) and other landmarks (e.g. coffee point) $(\phi=0.54, p<0.001$, and $0.39, p<0.05$, respectively). These results show that participants used different strategies for wayfinding: without navigation support they used signs and route colour, but with navigation support they used not only the artwork specifically installed to aid navigation, but also existing furniture and other landmarks.

Moreover, these strategies were linked to time-on-task: correlations of time-on-task on Tasks 1,2 , and 3 with the use of signs $(r=0.58,0.52$, and 0.57 , respectively, all $p$ $<0.01)$ and route colour $(r=0.32,0.32$, and 0.38 , respectively, all $p<0.05)$ were positive, but correlations were negative with use of existing furniture and other landmarks $(r=-0.46, p<0.01,-0.37$, and -0.32 , both $p<0.05)$. Therefore, relying on signs and route colour increased time-on-task, but using existing furniture and other landmarks decreased time-on-task. In addition, strategies were linked to errors on Task 1: the correlation with the use of signs was positive, $r=0.33, p<0.05$, but negative with the use of existing furniture and other landmarks, $r=-0.36, p<0.05$. Finally, time-on-task was linked to errors, $r=0.63, p<0.001$, and hesitations, $r=0.40, p=0.01$, on Task 1 , and with errors, $r=0.45, p<0.01$, on Task 3 .

Collectively, the results of these exploratory analyses together with those on strategy in relation to experimental condition and the use of artwork suggest that strategy is a determinant of accuracy of wayfinding, which in turn is a determinant of time-on-task (see also Section 5). 


\section{3-D route-planning support for wayfinding}

Although on their outward journey participants in the control condition did not know the artwork pieces and their location, it was observed that some used these as landmarks on their return journey. This observation supports the idea that people can quickly integrate landmarks in their internal representation of indoor environments and benefit from these in wayfinding.

Observations made during the tasks showed that Task 1 was the most difficult one. This was confirmed by post-hoc tests as follow-up of one-way repeated-measures analyses of variance with task as the independent variable, testing the effect of task on time-on-task ${ }^{3}$, disorientation ${ }^{4}$ and errors ${ }^{5}$ per condition. In the control condition, Task 1 was significantly higher than Tasks 2 and 3 on disorientation and error rate in (all $p \leq 0.001$ ) and lower on time-on-task (Task 1 - Task 2, $p<0.05$, Task 1 - Task $3, p<0.01$ ), but this was not the case in the experimental condition. ${ }^{6}$ Further evidence for Task 1 being the most difficult was a significant negative correlation of spatial ability with number of hesitations on this task, $r=-0.37, p<0.05$ (as well as a negative correlation with number of errors, $r=-0.25, p=0.11$ ). In other words, those with poorer spatial ability experienced greater difficulty on Task 1, but Tasks 2 and 3 were not sensitive to participants' individual differences in spatial ability. In Task 1, the following were common (indications of) errors in wayfinding: wrong turns, losing the way and going round in circles. Errors seemed to be due to the following causes: participants did not check the route by its colour in the corridors (orange, blue, green and yellow) or the level of the current location (ground, first or second

$3 \quad F(2,38)=10.23, p<0.001$ for the control condition, but $F<1$ for the experimental condition.

$4 F(2,38)=17.25, p<0.001$ for the control condition, but $F(2,38)=2.25, p>0.05$ for the experimental condition.

$5 \quad F(2,38)=20.03, p<0.001$ for the control condition, but $F(2,38)=3.25, p=0.05$ for the experimental condition.

6 Tasks 2 and 3 did not differ significantly. 


\section{3-D route-planning support for wayfinding}

floor), depended only on the provided signs although these were not sufficient for finding the destination, depended only on the signs although at some point (indicated by the [green] dot in Figure A1) the destination was not indicated on the signs any further, and did not pay attention to some available maps along the main corridor in the hospital. These problems were overcome in the experimental condition as participants were able to locate and orientate themselves using landmarks such as artwork (installed specifically for the purpose of the experiment), existing furniture (e.g. a sofa) and other landmarks (e.g. coffee point).

According to observations made during Task 3 , this was the second-most difficult task, with the following common errors in wayfinding: wrong turns and mostly passing the destination. These errors appeared to be due to the following causes. The signs leading to the destination were inconsistent in that they differed in terms of stating the full name of the destination or only an abbreviated name. Errors were also made because some destinations had similar abbreviations (e.g. CCU), thereby misleading participants, and participants checking neither floor nor colour of the destination's route. These problems were overcome in the experimental condition as the route-planning support system showed participants to first go to the first floor before showing the remainder of the route. Additionally, because the route used by the route-planning system had many several turns (five), participants seemed to depend on artwork placed at the end of some corridors before making any turn. Finally, although reaching the destination was facilitated in the experimental condition, a few participants passed the destination, which forced some of them to go back to the artwork they saw at the last turn (indicated by [red] dot in Figure A3). 
3-D route-planning support for wayfinding

According to observations made during Task 2, this was the least difficult task, but with the following common errors in wayfinding: passing the destination and making wrong turns. These errors seemed to be due participants taking apparently random ways to reach the destination without using either the route colour or the floor where the destination was located.

\subsection{The acceptance of 3-D route-planning support}

This section presents results related to Research Question 2. In the experimental condition, acceptance of the 3-D route-planning support system was high ${ }^{7}$, in terms of perceived usefulness $\left(\right.$ mean $=6.20 ; S D=0.19 ; C I_{\text {mean, } 0.95=[5.80 ; 6.60]}$ ), perceived ease of use $\left(\right.$ mean $\left.=6.44 ; S D=0.16 ; \mathrm{CI}_{\text {mean, } 0.95}=[6.10 ; 6.78]\right)$ and intention to use $($ mean $=6.22 ; S D=0.22 ;$ Clmean, $0.95=[5.75 ; 6.68])$. Moreover, all three acceptance measures were substantially negatively correlated with disorientation on each of the three routes $(-0.49<r<-0.73,0.05<p \leq 0.001)$. Therefore, the better participants' wayfinding experience (the less disorientation) was, the higher their acceptance was.

Overall, participants commented positively on the system in terms of the quality of the navigation interface, ease of use and clarity of representation showing the artwork, furniture and other landmarks in the hospital, which aided in recalling the route to each destination. However, some participants also reported that the system showed each route too fast for them to learn the route to be followed. Furthermore, the arrangement of furniture within the hospital was not always consistent with that shown by the route-planning support system. For example, some remembered particular furniture items from the route-planning support system to take a right turn

7 All confidence intervals exceeded the neutral value of 4 on the 7-point scales that were used. 


\section{3-D route-planning support for wayfinding}

indicated by [blue] dot in Figure A2. However, as some furniture was missing or new furniture had been added in the building, these participants skipped the right turn and ended up taking a different route, which also took them to the destination but caused further errors and confusion. In addition, some destinations (e.g. in Task 2) could have been reached following a shorter and easier route than shown by the system, which was a potential disadvantage for participants who were under the experimental condition.

\section{Discussion}

The current study is unique in terms of establishing the effect of 3-D route-planning support on wayfinding in a large complex public indoor environment using multiple routes, the role of landmarks and system acceptance. The fact that the research was conducted in a 'live'large hospital during normal operation with many staff, many patients and many visitors adds to the ecological validity of this work. In relation to Research Question 1, the results show an advantage of the use of 3-D route-planning support. In particular, this support proved useful in terms of reducing time-on-task on all routes, and errors and disorientation when the route was difficult, helping participants to successfully use landmarks to find their way and reducing anxiety. The importance and usefulness of the facilitating effect of route-planning support is further underlined by the very large effect sizes demonstrating the advantage. According to our results, the main reason for the advantage of routeplanning support is that it facilitated people's use of landmarks. Almost all participants navigating in the experimental condition (with route-planning support) reported using landmarks, but hardly any participants in the control condition (without route-planning support) did. Furthermore, the reported use of landmarks was 


\section{3-D route-planning support for wayfinding}

associated with reduced error rate, disorientation and anxiety. Moreover, participants used different strategies for wayfinding: without navigation support they used signs and route colour, but with navigation support they used not only the artwork specifically installed to aid navigation, but also existing furniture and other landmarks. Therefore, route-planning support appeared to facilitate the acquisition of landmark knowledge (of artwork as an experimental manipulation, furniture and other existing landmarks) as part of an internal spatial representation and as a basis for wayfinding (however, it was not the aim of this study to test this conjecture; see also Section 5). Our empirical results regarding route navigation difficulty (presented in Section 3.1) are consistent with our objective analysis of route navigation difficulty, highlighting the importance of the number of choice points (presented in Section 2.3). Moreover, route-planning support resulted in better outcomes, with significant effects for the more difficult route. This result indicates that, apart from its potential role in aiding the development of landmark knowledge, support is especially important when existing wayfinding support is insufficient or suboptimal.

In relation to Research Question 2, we found evidence for a high level of acceptance of 3-D route-planning support. This was true for perceived ease of use, perceived usefulness and intention to use. Moreover, when disorientation was lower, acceptance on each of these three measures was higher. Although disorientation was not experimentally manipulated, it seems plausible that a reduction in disorientation contributes to increased acceptance of 3-D route-planning support in wayfinding, as occurs in other domains (Saadé \& Otrakji, 2007). Participants' positive comments regarding ease of use and usefulness further strengthen these results. However, participants also noted shortcomings, indicating potential for improvement (see Section 5). 


\section{3-D route-planning support for wayfinding}

From our discussion in Section 1, the underlying idea of the 3-D route-planning system is that wayfinding can be improved by facilitating the development of an internal representation (cognitive map) of a complex physical environment by providing people with a preview of the route to a particular destination. In particular, the system should be useful in forming route knowledge, thereby aiding wayfinding throughout the route, which should then be useful on future occasions when wayfinding involves the same or similar routes. The system should also facilitate forming landmark knowledge; in this respect, appropriately designed conspicuous landmarks in addition to existing landmarks in the physical environment can be especially useful (see also Author Citation 2), which the system also shows during route-planning.

As discussed in Section 1, it is important to consider the three elements of the wayfinding system: people, design elements and operational elements. Regarding people, this research has focused on navigation and route-planning in able-bodied people. Although the necessity of building and using an internal representation (cognitive map) of the external environment for successful wayfinding applies equally to all people, people with disabilities may require specific types of support. For example, route-planning support for people with cognitive impairments (Chang \& Wang; 2010; Magableh \& Barrett, 2011) has been developed as well as wayfinding support for visually impaired people (Petrie et al., 1996; Coughlan \& Manduchi, 2009; Swobodzinski \& Raubal, 2009).

On the subject of design elements, the current research studied a large hospital. However, the approach taken here should apply equally to complex indoor environments in other domains, such as education (e.g. higher education; Münzer \& 
3-D route-planning support for wayfinding

Stahl, 2011), retail (e.g. shopping centres; Hower, 1999), travel (e.g. railway stations; Boersema \& Zwaga, 1985) and art (e.g. concert halls and multi-functional buildings). As regards operational elements, the current research examined route-planning support. Although the current study does not compare different approaches to aid wayfinding, the approach of route-planning support differs fundamentally from that of online navigation systems (e.g. GPS-based car navigation systems; Lavie \& OronGilad, 2013) or human operators. Online systems give directions as and when needed, but do not necessarily facilitate the development of an internal representation that would be useful in the future, whereas route-planning support is to promote the development of landmark and route knowledge. Given this fundamental difference, route-planning support and online navigation support may be complementary in facilitating navigation in complex environments and should be developed in conjunction.

\section{Future work and implications}

There are some potential limitations of the current study that may be addressed and could form as starting points for future research (as discussed later in this section). First, although this was not an aim of the research, the idea that the quality of internal spatial representation (cognitive map) is a mechanism for improved navigation was not explicitly tested. Second, although this was not an aim of the research, the idea that the quality of internal spatial representation (cognitive map) is a mechanism for improved navigation was not explicitly tested. Third, although the 3-D route planning support proved to be advantageous, the research did not test what might be the best presentation speed. Fourth, the research used able-bodied participants to reduce unwanted variability that might obscure any advantage of 3-D 
3-D route-planning support for wayfinding

route-planning support, so they results may not generalise to people with disabilities.

Fifth, although this was not an aim of the research, the causal link between

disorientation and acceptance was not experimentally tested.

The study reported here has highlighted several opportunities for future research.

Although the idea that the quality of internal spatial representation (cognitive map) is a mechanism for improved navigation was not explicitly tested, our study tested and provided evidence for the effect of route-planning condition on task performance, disorientation, anxiety and use of landmarks, demonstrating the value of 3-D routeplanning support. Future research would test people's knowledge of the route to their destination after planning and after actual navigation. This work would clarify how planning and navigation support the development of an internal spatial representation, in particular landmark knowledge and route knowledge. Route knowledge could be facilitated further through the presentation of symbolic information showing each turn on a route (e.g. with an arrow; Münzer \& Stahl, 2011). In addition, future work may study evidence for a general model of navigation (e.g. Jul \& Furnas, cited in Darken \& Peterson, 2002) to gain detailed knowledge of the navigation process. Furthermore, based on our findings, the research may also experimentally test the conjecture that wayfinding strategy is a determinant of accuracy of wayfinding, which in turn is a determinant of wayfinding speed. This work may be helpful in establishing how 3-D route-planning support facilitates specific aspects of wayfinding and may further inform the design of this support.

Moreover, future work may could investigate the relative merits of route-planning support and online navigation support in promoting the development of cognitive maps and study how these two can complement each other in wayfinding. An 
3-D route-planning support for wayfinding

\section{experimental design would compare each of these two types of support, the}

\section{combination of these and a control condition.}

It was noted in the current study that walking speed of the 3-D animation (set at 9.36 $\mathrm{km}$ per hour for efficient use of route-planning time, but too fast according to some participants) might be subject to improvement. Relevant to this is Cornell and Heth's (2000) discussion of the role of landmarks in the development of a cognitive map. In particular, "to be a landmark, an object must be part of a spatial relationship; a landmark is always defined in relation to another object or frame of reference" ( $p$. 70). In addition, "the ability to register what is peripheral allows a place to be known with more area" (p. 70). However, enough time is needed to encode neighbouring objects of landmarks and, if not enough time is available, this process may not or only partially be completed. Moreover, people differ in their rate of encoding. For example, those who are slow at encoding (e.g. children) may be poor at recognising places and navigation (Cornell \& Heth, 2000). The implications are that, first, 3-D animations must allow sufficient time for encoding and, second, because people differ in encoding speed, should allow people to select the speed of animation.

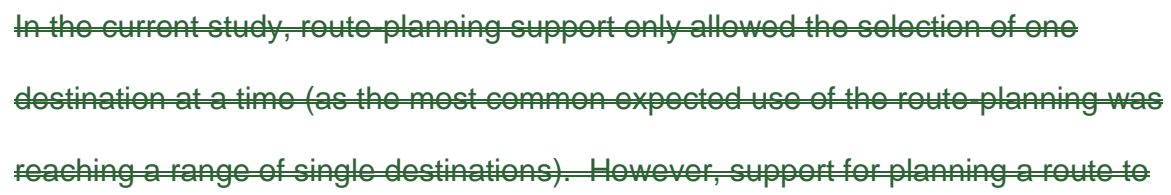


3-D route-planning support for wayfinding

time the system is consulted. Although the flexibility offered by route planning
support for multiple destinations seems attractive, in practice it could result in

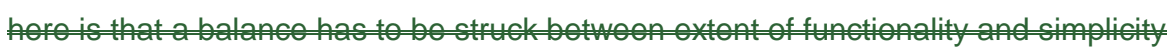
(Norman, 2011).

The location of the route-planning support system within a complex building's architectural design stage should also be considered. In other words, the arrangement of departments within a complex building can play an important role in

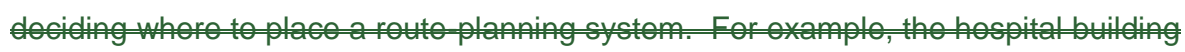
studied in the current research had nine entrances. The implication is that routeplanning support would have to be provided from each of these, with nine different rotes to destination in building.

Our participants were able-bodied students, as the purpose here was to examine if route-planning support yielded its facilitating effect on wayfinding, without complicating the experimental design with variables and confounds due to person characteristics; however, future research could be aimed at replicating the findings for specialised user groups. There are specific issues in route-planning for people with special needs. First, although route-planning systems usually rely on visual representations, these are normally not suitable for visually impaired people, but other representations may be useful (Petrie et al., 1996). Audio (e.g. narration) and tactile representations can be effective, but this still needs to be demonstrated in indoor wayfinding. Second, people with particular disabilities may need different routes (Fallah et al., 2013), for example avoiding ramps or stairs in the case of wheelchair users, for instance in favour of the use of lifts. The implications are that 
3-D route-planning support for wayfinding

route-planning systems thus need to be flexible, first, in the type of representations of the physical environment that they present and, second, in terms of constraints on the route to a particular destination. Third, (normally silent) landmarks can pose a problem for visually impaired people in that they will normally not notice these, although landmarks could help them in building a cognitive map to use in their wayfinding. As an implication, one solution would be augmenting a landmark with sound when approached in the physical world (as already happens at pedestrian crossings in the outdoor world), which would be matched by a sound in the presented route by the system during route-planning.

Given that reduced disorientation was linked to increase acceptance (see also Saadé \& Otrakji, 2007), our results motivate further research to experimentally test a causal link. For example, different versions of a 3-D route-planning support system may offer different levels of support in building (more or less complete) cognitivespatial representations, thereby resulting in different levels of (less or more) disorientation. It becomes then possible to test whether disorientation acts as a mediator of the effect of degree of support on acceptance.

In the current study, route-planning support only allowed the selection of one destination at a time (as the most common expected use of the route-planning was reaching a range of single destinations). However, support for planning a route to two or more destinations could be valuable in particular situations (e.g. when people plan a route to several shops in the same shopping centre). For instance, when deciding to go to Destination A followed by Destination B, the route-planning system would show the route to from a starting point to Destination A and then the route to Destination B from Destination A rather than the route to a single destination each 
3-D route-planning support for wayfinding

time the system is consulted. Although the flexibility offered by route-planning support for multiple destinations seems attractive, in practice it could result in complex system that may be counterproductive and less effective. The implication here is that a balance has to be struck between extent of functionality and simplicity (Norman, 2011).

The location of the route-planning support system within a complex building's architectural design stage should also be considered. In other words, the arrangement of departments within a complex building can play an important role in deciding where to place a route-planning system. For example, the hospital building studied in the current research had nine entrances. The implication is that routeplanning support would have to be provided from each of these, with nine different routes to each destination in the building.

\section{Conclusion}

In conclusion, this empirical study has identified several benefits of 3-D routeplanning support in complex public buildings; these include improving people's navigation performance, reducing disorientation and anxiety, and promoting a landmark-based wayfinding strategy. Moreover, this support was well accepted. It is therefore important that organisations in health-care and other sectors exploit the concept of 3-D route-planning and benefit from it, while at the same time taking into account other elements of the wayfinding system. 
3-D route-planning support for wayfinding

\section{Acknowledgement}

The authors are grateful to ***** (name of establishment removed to facilitate masked review) for adapting the 3-D application for the research and making the artwork available. The authors are also grateful to **** (name of establishment removed to facilitate masked review) hospital for operational support.

\section{References}

Author Citation 1

Author Citation 2

ABED M.A., HALL L.A. and MOSER D.K., 2011, Spielberger's State Anxiety Inventory: Development of a shortened version for critically III patients. Issues in Mental Health Nursing, 32, pp. 220-227.

BOERSEMA T. and ZWAGA H.J.G., 1985, The influence of advertisements on the conspicuity of routing information. Applied Ergonomics, 16, pp. 267-273.

CARPMAN J. and GRANT M., 2002, Wayfinding: a broad view. In Handbook of environmental psychology, R. BECHTEL and A. CHURCHMAN (Eds.), pp. 427-442 (New York: Wiley).

CHANG Y.= and WANG T.=, 2010, Comparing picture and video prompting in autonomous indoor wayfinding for individuals with cognitive impairments. Personal and Ubiquitous Computing, 14, pp. 737-747. 
3-D route-planning support for wayfinding

COHEN J., 1988, Statistical power analysis for the behavioral sciences (Hillsdale, NJ: Erlbaum).

CORNELL E. and HETH D., 2000, Route learning and wayfinding. In Cognitive mapping: past, present and future, ROB KITCHIN and SCOTT FREUNSCHUH (Eds.), pp. 66-83 (New York: Routledge).

COUGHLAN J. and MANDUCHI R., 2009, Functional assessment of a camera phone-based wayfinding system operated by blind and visually impaired users. International Journal on Artificial Intelligence Tools, 18, pp. 379-397.

DARKEN R. and PETERSON B., 2002, Spatial orientation, wayfinding, and representation. In Handbook of virtual environments, K. STANNEY (Ed.), pp. 493518 (Mahwah, NJ: Erlbaum).

\section{DARKEN R.P. and SIBERT J.L., 1996, Navigating Large Virtual}

Spaces. International Journal of Human-Computer Interaction, 8, pp. 49-71.DOWNS

Harper and Row).

FALLAH N., APOSTOLOPOULOS I., BEKRIS K. and FOLMER E., 2013, Indoor human navigation systems: a survey. Interacting with Computers, 25, pp. 21-33.

FRANKENSTEIN J., BRÜSSOW S., RUZZOLI F. and HÖLSCHER C., 2012, The language of landmarks: the role of background knowledge in indoor wayfinding. Cognitive Processing, 23, pp. 1-6. 
3-D route-planning support for wayfinding

GEORGE SAADÉ R. and ALEXANDRE OTRAKJI C., 2007, First impressions last a lifetime: effect of interface type on disorientation and cognitive load. Computers in Human Behavior, 23, pp. 525-535.

GOODMAN J., BREWSTER S.A. and GRAY P., 2005, How can we best use landmarks to support older people in navigation? Behaviour and Information Technology, 24, pp. 3-20.

HOWER B.K., 1999, Finding the way. Industrial Fabric Products Review, 76, pp. 1619.

JUL S. and FURNAS G., 1997, Navigation in electronic worlds: a CHI 97 workshop. SIGCHI Bulletin, 29(4), pp. 44-49.

LAVIE T. and ORON-GILAD T., 2013, Perceptions of electronic navigation displays. Behaviour and Information Technology, 32, pp. 800-823.

LAWTON C.A., CHARLESTON S.I. and ZIELES A.S., 1996, Individual- and genderrelated differences in indoor wayfinding. Environment and Behavior, 28, pp. 204-219.

MAGABLEH B. and BARRETT S., 2011, Self-adaptive application for indoor wayfinding for individuals with cognitive impairments. In 24th International Symposium on Computer-Based Medical Systems, CBMS 2011, 27 June 2011 through 30 June 2011, Bristol.

MÜNZER S. and STAHL C., 2011, Learning Routes from Visualizations for Indoor Wayfinding: Presentation Modes and Individual Differences. Spatial Cognition and Computation, 11, pp. 281-312. 
3-D route-planning support for wayfinding

NIELSEN J. and LEVY J., 1994, Measuring usability preference vs.

performance. Communications of the ACM, 37, pp. 66-75.

NORMAN D., 2011, Living with complexity (Cambridge, MA: MIT Press).

PADGITT A.J. and HUND A.M., 2012, How good are these directions? Determining direction quality and wayfinding efficiency. Journal of Environmental Psychology, 32, pp. 164-172.

PADGITT A.J. and HUND A.M., 2012, How good are these directions? Determining direction quality and wayfinding efficiency. Journal of Environmental Psychology, 32 , pp. 164-172.

PETRIE H., JOHNSON V., STROTHOTTE T., RAAB A., FRITZ S. and MICHEL R., 1996, MOBIC : Designing a travel aid for blind and elderly people. Journal of Navigation, 49, pp. 45-52.

RIENTIES B., GIESBERS B., LYGO-BAKER S., MA H.W.S. and REES R., 2014, Why some teachers easily learn to use a new virtual learning environment: a technology acceptance perspective. Interactive Learning Environments, .

ROOKE C., TZORTZOPOULOS P., KOSKELA L. and ROOKE J., 2009, Wayfinding: embedding knowledge in hospital environments. In HaCIRIC 2009: Improving Healthcare Infrastructure Through Innovation_Anonymous (Brighton: Hilton Metropole).

SPIELBERGER C.D., 1983, Manual for the State-trait Anxiety Inventory STAI (form Y) ('Self-Evaluation Questionnaire') (California: Consulting Psychology Press). 
3-D route-planning support for wayfinding

STANKIEWICZ B.J., LEGGE G.E. and SCHLICHT E., 2001, The effect of layout complexity on human and ideal navigation performance. Journal of Vision, 1.

SWOBODZINSKI M. and RAUBAL M., 2009, An indoor routing algorithm for the blind: Development and comparison to a routing algorithm for the sighted. International Journal of Geographical Information Science, 23, pp. 13151343.

TOLMAN E.C., 1948, Cognitive maps in rats and men. Psychological Review, 55,

pp. $189-208$.

VANDENBERG S. and KUSE A., 1978, Mental rotations, a group test of threedimensional spatial visualization. Perceptual and Motor Skills, 47, pp. 599-604.

VENKATESH V., DAVIS F.D. and MORRIS M.G., 2007, Dead or alive? The development, trajectory and future of technology adoption research. Journal of the Association of Information Systems, 8, pp. 267-286.

VENKATESH V., MORRIS M.G., DAVIS G.B. and DAVIS F.D., 2003, User acceptance of information technology: Toward a unified view. MIS Quarterly: Management Information Systems, 27, pp. 425-478.

VILAR E., REBELO F. and NORIEGA P., 2012, Indoor human wayfinding performance using vertical and horizontal signage in virtual reality. Human Factors and Ergonomics in Manufacturing, 19.

VILAR E., REBELO F., NORIEGA P., TELES J. and MAYHORN C., 2013, The influence of environmental features on route selection in an emergency situation. Applied Ergonomics, 44, pp. 618-627. 
3-D route-planning support for wayfinding

WRIGHT P., SOROKA A., BELT S., PHAM D.T., DIMOV S., DE ROURE D. and PETRIE H., 2010, Using audio to support animated route information in a hospital touch-screen kiosk. Computers in Human Behavior, 26, pp. 753-759.

XIE H., FILIPPIDIS L., GALEA E.R., BLACKSHIELDS D. and LAWRENCE P.J., 2012, Experimental analysis of the effectiveness of emergency signage and its implementation in evacuation simulation. Fire and Materials, 36, pp. 367-382.

XU L., HUANG B. and TANG Z., 2009, Spatial differentiation effect on wayfinding and orientation in gestalt space. Tongji Daxue Xuebao/Journal of Tongji University, 37, pp. 148-154. 
3-D route-planning support for wayfinding

Table 1

Descriptives by route-planning-support condition

\begin{tabular}{|c|c|c|}
\hline & Experimental & Control \\
\hline \multirow[t]{2}{*}{ Pre-test anxiety } & 3.30 & 3.37 \\
\hline & $(0.17)$ & $(0.13)$ \\
\hline \multirow[t]{2}{*}{ Post-test anxiety } & 2.85 & 3.63 \\
\hline & $(0.26)$ & $(0.21)$ \\
\hline \multirow[t]{2}{*}{ Time-on-task, Task 1} & 5.60 & 7.75 \\
\hline & $(0.17)$ & $(0.18)$ \\
\hline \multirow{2}{*}{ Time-on-task, Task 2} & 5.65 & 7.00 \\
\hline & $(0.17)$ & $(0.15)$ \\
\hline \multirow[t]{2}{*}{ Time-on-task, Task 3} & 5.65 & 6.80 \\
\hline & $(0.21)$ & $(0.12)$ \\
\hline \multirow[t]{2}{*}{ Disorientation, Task 1} & 1.63 & 2.95 \\
\hline & $(0.21)$ & $(0.35)$ \\
\hline \multirow[t]{2}{*}{ Disorientation, Task 2} & 1.82 & 1.88 \\
\hline & $(0.36)$ & $(0.31)$ \\
\hline \multirow[t]{2}{*}{ Disorientation, Task 3} & 1.48 & 2.08 \\
\hline & $(0.28)$ & $(0.32)$ \\
\hline \multirow[t]{2}{*}{ Errors, Task 1} & 0.50 & 2.10 \\
\hline & $(0.22)$ & $(0.32)$ \\
\hline \multirow[t]{2}{*}{ Errors, Task 2} & 0.30 & 0.35 \\
\hline & $(0.18)$ & $(0.13)$ \\
\hline \multirow[t]{2}{*}{ Errors, Task 3} & 0.00 & 0.50 \\
\hline & $(0.00)$ & $(0.18)$ \\
\hline \multirow[t]{2}{*}{ Hesitations, Task 1} & 0.35 & 0.90 \\
\hline & $(0.17)$ & $(0.23)$ \\
\hline \multirow[t]{2}{*}{ Hesitations, Task 2} & 0.05 & 0.15 \\
\hline & $(0.05)$ & $(0.11)$ \\
\hline \multirow[t]{2}{*}{ Hesitations, Task 3} & 0.15 & 0.05 \\
\hline & $(0.08)$ & $(0.05)$ \\
\hline
\end{tabular}

Note. Figures are means, with SE in brackets. Results per task are for the outward journey. 
3-D route-planning support for wayfinding

Table 2

Descriptives by reported artwork use

\begin{tabular}{|c|c|c|}
\hline & $\begin{array}{l}\text { Artwork } \\
\text { not used }\end{array}$ & $\begin{array}{l}\text { Artwork } \\
\text { used }\end{array}$ \\
\hline \multirow[t]{2}{*}{ Pre-test anxiety } & 3.30 & 3.37 \\
\hline & $(0.18)$ & $(0.12)$ \\
\hline \multirow[t]{2}{*}{ Post-test anxiety } & 3.54 & 2.97 \\
\hline & $(0.26)$ & $(0.23)$ \\
\hline \multirow[t]{2}{*}{ Time-on-task, Task 1} & 7.63 & 5.81 \\
\hline & $(0.23)$ & $(0.20)$ \\
\hline \multirow[t]{2}{*}{ Time-on-task, Task 2} & 6.89 & 5.81 \\
\hline & $(0.15)$ & $(0.20)$ \\
\hline \multirow[t]{2}{*}{ Time-on-task, Task 3} & 6.79 & 5.71 \\
\hline & $(0.12)$ & $(0.21)$ \\
\hline \multirow[t]{2}{*}{ Disorientation, Task 1} & 3.00 & 1.65 \\
\hline & $(0.37)$ & $(0.20)$ \\
\hline \multirow[t]{2}{*}{ Disorientation, Task 2} & 1.96 & 1.75 \\
\hline & $(0.32)$ & $(0.34)$ \\
\hline \multirow[t]{2}{*}{ Disorientation, Task 3} & 2.09 & 1.51 \\
\hline & $(0.33)$ & $(0.27)$ \\
\hline \multirow[t]{2}{*}{ Errors, Task 1} & 2.00 & 0.67 \\
\hline & $(0.35)$ & $(0.24)$ \\
\hline \multirow[t]{2}{*}{ Errors, Task 2} & 0.37 & 0.29 \\
\hline & $(0.14)$ & $(0.17)$ \\
\hline \multirow[t]{2}{*}{ Errors, Task 3} & 0.47 & 0.05 \\
\hline & $(0.19)$ & $(0.05)$ \\
\hline \multirow[t]{2}{*}{ Hesitations, Task 1} & 0.89 & 0.38 \\
\hline & $(0.24)$ & $(0.16)$ \\
\hline \multirow[t]{2}{*}{ Hesitations, Task 2} & 0.05 & 0.14 \\
\hline & $(0.05)$ & $(0.10)$ \\
\hline \multirow[t]{2}{*}{ Hesitations, Task 3} & 0.16 & 0.05 \\
\hline & $(0.09)$ & $(0.05)$ \\
\hline
\end{tabular}

Note. Figures are means, with SE in brackets. Results per task are for the outward journey. 
3-D route-planning support for wayfinding

\section{Figure captions}

Figure 1. LLtandmarks: that were strategically placed in the hospital corridors at choice points to facilitate wayfinding.

Figure 2. Screenshots Representation of hospital environment represented in 3-D route-planning system with landmarks at choice points to facilitate wayfinding.- 
3-D route-planning support for wayfinding
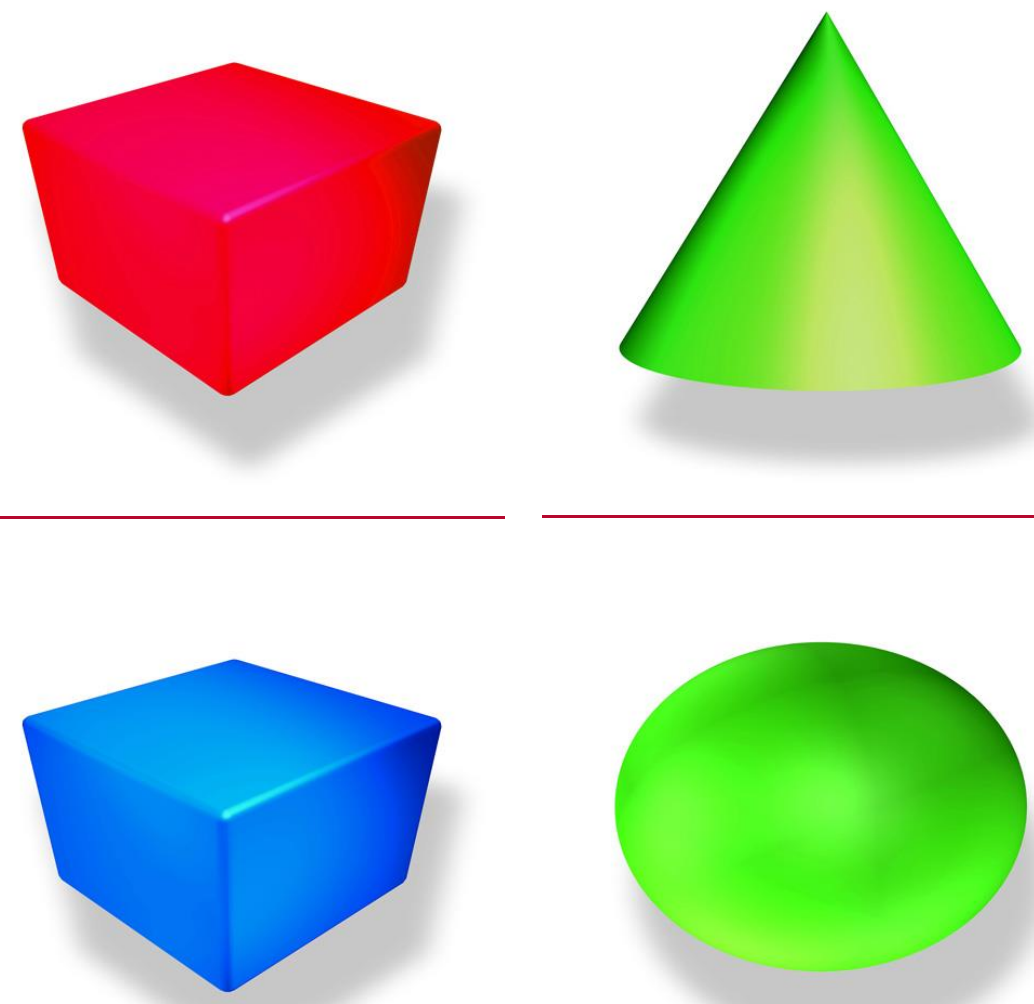

Figure 1.

Formatted: Line spacing: Double 
3-D route-planning support for wayfinding
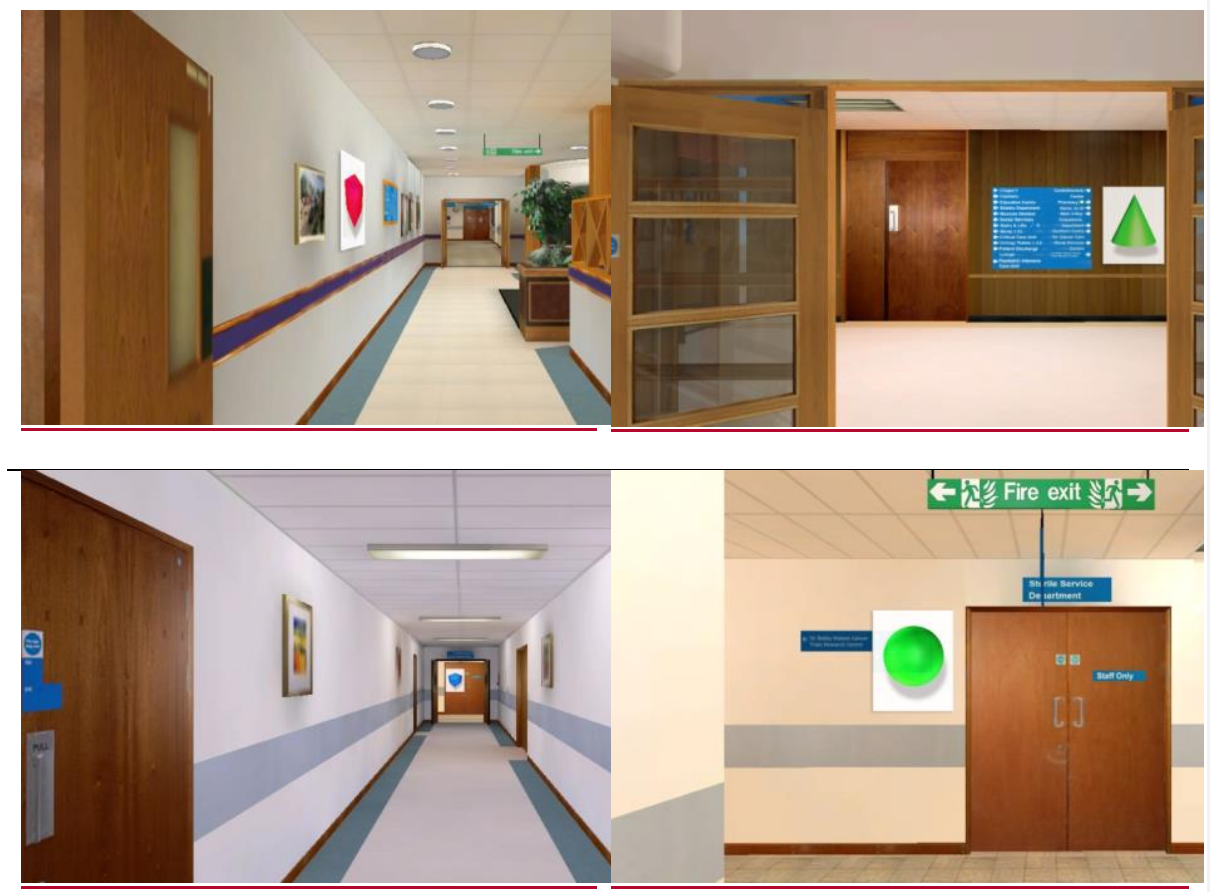

Figure 2 
3-D route-planning support for wayfinding

\section{Appendix: routes}

Figure captions

Figure A1. Task 1: South Entrance - Ward 16.

Figure A2. Task 2: South Entrance-CCU.

Figure A3. Task 3: South Entrance-CICU. 